\title{
Clinical applications of therapeutic phlebotomy
}

This article was published in the following Dove Press journal:

Journal of Blood Medicine

18 July 2016

Number of times this article has been viewed

\section{Kyung Hee Kim' \\ $\mathrm{Ki}$ Young $\mathrm{Oh}^{2}$}

'Department of Laboratory Medicine, Gachon University Gil Medical

Center, Incheon, ${ }^{2}$ Department of Physical Medicine and Rehabilitation, Soonchunhyang University, Cheonan

Hospital, Cheonan, South Korea
Correspondence: Ki Young Oh Department of Physical Medicine and Rehabilitation, Soonchunhyang University Cheonan Hospital,

Bongmyeong-dong, Dongnam-gu, Cheonan-si, Chungcheongnam-do 330-721, South Korea

Tel +82 4I 5703884

Fax +82 4I 5702776

Email okokykkh@schmc.ac.kr
Abstract: Phlebotomy is the removal of blood from the body, and therapeutic phlebotomy is the preferred treatment for blood disorders in which the removal of red blood cells or serum iron is the most efficient method for managing the symptoms and complications. Therapeutic phlebotomy is currently indicated for the treatment of hemochromatosis, polycythemia vera, porphyria cutanea tarda, sickle cell disease, and nonalcoholic fatty liver disease with hyperferritinemia. This review discusses therapeutic phlebotomy and the related disorders and also offers guidelines for establishing a therapeutic phlebotomy program.

Keywords: therapeutic phlebotomy, hemochromatosis, polycythemia vera, porphyria cutanea tarda, sickle cell disease, nonalcoholic fatty liver disease

\section{Introduction}

Phlebotomy is also known as bloodletting or venesection and is an important treatment that has been used by various groups from ancient times to the present. ${ }^{1,2}$ Phlebotomy has historically been performed using cupping, acupuncture, or leeches, ${ }^{2}$ although these procedures occasionally lead to death. One famous example is George Washington (former President of the US), who died after losing $1.7 \mathrm{~L}$ of blood during treatment for acute epiglottitis. ${ }^{3}$

Phlebotomy using inserted needles differs from wet cupping therapy (Al-hijamah). ${ }^{4}$ During Al-hijamah, local suction is created at the selected areas using special cups, which are subsequently removed after several minutes. The skin is then scratched using a sterile cupping scalpel, and then the cups are replaced to carefully draw out small quantities of blood and remove toxins. Cupping therapy (alone or with other interventions) may be beneficial for painful conditions, such as herpes zoster, acne, facial paralysis, cervical spondylosis, rheumatoid arthritis, brachialgia paraesthetica nocturna, carpal tunnel syndrome, acute gouty arthritis, fibrositis, fibromyalgia, persistent nonspecific lower back pain, acute trigeminal neuralgia, headaches, and migraines. ${ }^{4-6}$ Several thousand years ago, phlebotomy was used for both preventative and curative treatments. However, in modern medicine, phlebotomy is performed in a physician's office, at a blood bank, or under the supervision of a doctor in a hospital and is only performed with a prescription that clearly specifies the clinical indication(s) and the required number of bloodlettings. ${ }^{7}$

Therapeutic phlebotomy has several physiological mechanisms. ${ }^{8}$ For example, bone marrow stem cells are stimulated by bloodletting to generate new red blood cells 
(RBCs), which requires the transport of iron (in the ferritin form) from the body's stores to create hemoglobin (Hb). Thus, the patient's overall iron levels are reduced, which makes therapeutic phlebotomy the preferred treatment for blood disorders in which the removal of RBCs or serum iron is the most efficient method for managing the symptoms and complications. ${ }^{9}$ There are currently three major indications for therapeutic phlebotomy: hemochromatosis, polycythemia vera, and porphyria cutanea tarda. Other indications include sickle cell disease and nonalcoholic fatty liver disease (NAFLD) with hyperferritinemia.

In this review, we examine the major indications for therapeutic phlebotomy and provide guidelines for phlebotomy, its effects and specific treatments, and additional information that may help facilitate the use of therapeutic phlebotomy for South Korean patients. Moreover, we provide recommendations for the creation of new therapeutic phlebotomy programs.

\section{Indications for therapeutic phlebotomy Polycythemia vera}

Polycythemia vera is a clonal progressive myeloproliferative disorder that is associated with significant erythrocytosis and is characterized by the increased bone marrow production of erythrocytes, which leads to an increase in their numbers and higher blood viscosity. ${ }^{10}$ The production of white blood cells and platelets can also be increased. Therapeutic phlebotomy is the best choice for initial therapy, and one of its key treatment objectives is to reduce the risk of these thrombotic events. ${ }^{8}$ This is because patients with polycythemia vera have an increased risk of thrombotic events, such as cerebrovascular disease, cardiovascular disease, and arterial or venous thromboembolism. Previous studies regarding polycythemia vera have indicated that patients who received phlebotomy exhibited a lower incidence of hematomas and solid tumors, and other studies have reported that phlebotomy-induced maintenance of target hematocrit levels $(<45 \%)$ was associated with significantly lower cardiovascular morbidity and a lower incidence of major thrombotic diseases. ${ }^{11,12}$

Phlebotomy can also be performed in hypoxemic conditions, such as those that occur during chronic lung disease or hemochromatosis. Patients with hypoxemic lung disease who exhibit symptoms of hyperviscosity syndrome or have elevated hematocrit levels (>56\%) should undergo phlebotomy to reduce their hematocrit levels to $50 \%-52 \%{ }^{13-15}$ The American Heart Association also recommends therapeutic phlebotomy for cyanotic congenital heart disease that presents with $\mathrm{Hb}$ levels of $>20 \mathrm{~g} / \mathrm{dL}$ and hematocrit levels of $>65 \% .{ }^{16}$ Acquired iron overload because of repeated transfusions is another indication for therapeutic phlebotomy. In this context, patients who are receiving chronic blood transfusions (eg, for sickle cell anemia or thalassemia) may develop iron overload and iron deposition in various organs, which may lead to multiple organ failure. ${ }^{8}$

\section{Hemochromatosis}

Although phlebotomy does not clinically improve hemochromatosis, it can prevent complications in patients with symptoms or organ damage. ${ }^{17}$ Continuous phlebotomy is recommended until the patient's serum ferritin levels are $\leq 50 \mathrm{ng} / \mathrm{mL}$ and their transferrin saturation is $<50 \% .{ }^{18}$ Furthermore, the 2011 practice guidelines for hemochromatosis treatment from the American Association for the Study of Liver Diseases ${ }^{19}$ indicate that phlebotomy induces various therapeutic responses in hemochromatosis, which include the normalization of tissue iron levels, improved patient survival and cardiac function, and reduced abdominal pain and pigmentation.

\section{Porphyria cutanea tarda}

Porphyria cutanea tarda is a disease that is characterized by uroporphyrinogen accumulation, which is related to low levels of uroporphyrinogen decarboxylase. Therapeutic phlebotomy is the optimal treatment for this disease, and hydroxychloroquine treatment is a good alternative. ${ }^{20}$ In these cases, phlebotomy should be repeated every 2 weeks until the $\mathrm{Hb}$ levels are $<20 \mathrm{ng} / \mathrm{mL} .{ }^{21}$ Other studies have suggested that erythrocytapheresis is an alternative to phlebotomy. ${ }^{22}$

\section{Sickle cell disease}

Sickle cell disease (SS or SA variants) may benefit from phlebotomy alone or in combination with hydroxyurea. ${ }^{7}$ This is because phlebotomy decreases the blood's viscosity by reducing the $\mathrm{Hb}$ levels and the mean corpuscular $\mathrm{Hb}$ concentration, which subsequently reduces the $\mathrm{HbS}$ polymerization that is observed during sickle cell disease. ${ }^{23}$ Bouchaïr et al ${ }^{23}$ reported that frequent phlebotomies reduced hospitalization duration and lowered $\mathrm{Hb}$ levels without any adverse events, although only patients with high $\mathrm{Hb}$ levels were likely to benefit from phlebotomy. Rombos et $\mathrm{al}^{24}$ demonstrated that weekly phlebotomy improved the duration, frequency, and severity of pain crises and suggested that regular phlebotomies were associated with a considerable placebo effect.

The Stroke With Transfusions Changing to Hydroxyurea trial (SWiTCH, ClinicalTrials.gov NCT00122980) compared 
chronic blood transfusions and chelation to hydroxycarbamide (also known as hydroxyurea) and phlebotomy for recurrent stroke and iron overload management in children with sickle cell anemia and stroke. ${ }^{25}$ In that study, the transfusions were stopped and serial phlebotomy was started after the maximum tolerated dose of hydroxycarbamide was reached. Therapeutic phlebotomy was performed every $4 \pm 1$ weeks, and the initial procedure was performed at $5 \mathrm{~mL} / \mathrm{kg}$, although the subsequent phlebotomy volumes were increased to $10 \mathrm{~mL} / \mathrm{kg}$ with a maximum of $500 \mathrm{~mL}$ in cases with $\mathrm{Hb}$ levels of $\geq 8 \mathrm{~g} / \mathrm{dL}$. Interestingly, phlebotomy was only associated with self-limited adverse events (prevalence: $3.5 \%$ ), such as hypotension, dizziness, syncope, headache, and weakness. Furthermore, the SWiTCH trial demonstrated that the net iron balance was favorable, with a significant decrease in ferritin levels, and that phlebotomy was safe and well tolerated, with net iron removal in most children who completed 30 months of protocol-directed treatment.

Summarell and Sheehan ${ }^{26}$ recently evaluated the use of hydroxyurea and phlebotomy in hemoglobin SC (HbSC) disease. HbSC exhibits significantly different pathophysiology, compared to hemoglobin SS, as HbSC does not sickle and instead causes cellular dehydration that potentiates the sickling of hemoglobin SS. In that study, patients with HbSC who failed to achieve clinical improvement after receiving the maximum tolerated dose of hydroxyurea were offered phlebotomy and hydroxyurea, and all patients exhibited at least partial clinical improvement. Therefore, the authors suggested that dual therapy using hydroxyurea and phlebotomy may be an effective approach for patients with $\mathrm{HbSC}$ who do not experience improvement with hydroxyurea alone.

\section{NAFLD with hyperferritinemia}

NAFLD is the most common cause of chronic liver disease and is anticipated to become the most frequent indication for liver transplantation by $2030 .{ }^{27}$ NAFLD is defined as lipiddeposit accumulation in hepatocytes, which is not related to excessive alcohol consumption and is instead related to type 2 diabetes mellitus, insulin resistance (IR), central obesity, hyperlipidemia, and hypertension. ${ }^{28}$ NAFLD also encompasses nonalcoholic steatohepatitis, which can lead to liver fibrosis, cirrhosis, and hepatocellular carcinoma, ${ }^{27,29}$ and is defined as liver fat accumulation that is $>5 \%$ of its weight. ${ }^{30}$ Patients with NAFLD often exhibit increased hepatic iron deposition and elevated serum ferritin levels, although $20 \%$ $30 \%$ of NAFLD cases exhibit hyperferritinemia with mild hepatic iron accumulation, which is commonly referred to as dysmetabolic iron overload syndrome. ${ }^{28}$ Hyperferritinemia and increased iron stores have also been associated with hepatocellular carcinoma in patients with NAFLD. ${ }^{31,32}$

Valenti et $\mathrm{al}^{31}$ investigated the effects of phlebotomy in 38 patients with NAFLD who were randomized to undergo phlebotomy $(n=21)$ or lifestyle changes alone $(n=17)$. Phlebotomy was performed by removing $350 \mathrm{~mL}$ of blood every 10-15 days, and this procedure led to no adverse events. The main study outcome was improvement in liver damage after 2 years, according to the NAFLD activity scores. The secondary outcome was improvements in the levels of liver enzymes, including aspartate transaminase, alanine transaminase, and gamma-glutamyltransferase. Phlebotomy was associated with a normalization of iron parameters and an amelioration of liver enzyme levels. Compared to lifestyle changes alone, iron depletion via phlebotomy was associated with a higher rate of improvement in histological liver damage among patients with NAFLD and hyperferritinemia. However, conflicting results were observed in a more recent prospective randomized controlled trial that investigated the effect of phlebotomy with lifestyle advice among patients with NAFLD ${ }^{33}$ The primary end points were hepatic steatosis (as quantified using magnetic resonance imaging) and liver injury (as measured using levels of alanine transaminase and cytokeratin-18). The secondary end points were IR (as determined using the insulin sensitivity index and the homeostasis model of assessment) and systemic lipid peroxidation (plasma F2-isoprostane levels). That study found that phlebotomy reduced ferritin levels, but did not improve liver enzyme levels, hepatic fat, or IR. Therefore, larger trials are needed to investigate the therapeutic effects of phlebotomy for severe NAFLD/ nonalcoholic steatohepatitis and hyperferritinemia that is resistant to lifestyle changes.

\section{Non-phlebotomy depletion of RBCs and iron}

In addition to phlebotomy, there are several other options for reducing RBC and iron levels. One trial investigated erythrocyte apheresis instead of phlebotomy, ${ }^{9}$ based on the hypothesis that two- to threefold more iron would be removed per treatment, which would require less frequent phlebotomies. However, apheresis is associated with various limitations, including a high cost, longer treatment times, and various restrictions for treatment. Another treatment option is deferoxamine (an iron-chelating medication), which is capable of binding up to $85 \mathrm{mg}$ of iron per $1 \mathrm{~g}$ of medication; this treatment can reduce the iron load by $250 \mathrm{mg} / 500 \mathrm{~mL} .^{34}$ Deferoxamine may be beneficial for patients who cannot tolerate phlebotomy. The use of interferon as a myelosuppressive 
agent may also provide advantages for patients with polycythemia vera. ${ }^{9}$ Other medications that reduce the platelet count are associated with decreased risks of post-phlebotomy thrombosis, although they may also increase the risk of developing leukemia. ${ }^{9}$

\section{Points to consider for implementing therapeutic phlebotomy}

When a therapeutic phlebotomy program is introduced, the institution must establish guidelines and regulations for patient safety, the phlebotomy procedure, and blood disposal. One good source of related information is the practice criteria of the American Association of Blood Banks. ${ }^{35}$ Furthermore, regulations for phlebotomy are also provided in Standard 66 of Practice Criteria III from the Infusion Nursing Standards of Practice that were established by the Infusion Nurses Society. These regulations emphasize patient education regarding intra- and post-phlebotomy symptoms and consent, and include training for staff regarding how to manage adverse effects and infections, how to reach the target blood vessel, venous phlebotomy techniques, patient safety, and basic cardiopulmonary resuscitation. ${ }^{35}$

Patient factors, such as age, sex, weight, comorbidities, overall health status, and the likelihood of compliance, should be considered when prescribing a phlebotomy regimen. ${ }^{8}$ Zubair $^{8}$ has described various criteria for initiating therapeutic phlebotomy, which include ferritin levels of $>200 \mathrm{ng} / \mathrm{mL}$ for ages of $<18$ years (regardless of sex), ferritin levels of $>500 \mathrm{ng} / \mathrm{mL}$ for women who are not pregnant but are of childbearing age, and ferritin levels of $>300 \mathrm{ng} / \mathrm{mL}$ for men who are $>18$ years old. A half unit $(250 \mathrm{~mL})$ of blood can be removed during each session for patients with a small body mass, anemia, or cardiac or pulmonary disorders, and each unit of blood $(500 \mathrm{~mL})$ typically represents the removal of 200-250 mg of iron, depending on the Hb levels. ${ }^{8}$ In this context, serum ferritin and $\mathrm{Hb}$ levels are the most reliable measures for monitoring patients who are receiving therapeutic phlebotomy. Patients with significantly elevated initial ferritin levels $(\geq 1,000 \mathrm{ng} / \mathrm{mL})$ should have their serum ferritin levels monitored every 2-3 months, and $\mathrm{Hb}$ levels should be checked during each phlebotomy visit. This is because patients with a pre-phlebotomy $\mathrm{Hb}$ concentration of $<11 \mathrm{~g} / \mathrm{dL}$ are more likely to experience symptoms of hypovolemia and anemia, and therapeutic phlebotomy is less efficient in depleting iron stores at $\mathrm{Hb}$ levels of $<11 \mathrm{~g} / \mathrm{dL}{ }^{8}$

A doctor's prescription is required for therapeutic phlebotomy, as it is a medical intervention, ${ }^{9}$ and this prescription should indicate the frequency of the phlebotomy procedures and the target blood volume. Replacement fluids should also be prescribed for patients who cannot tolerate the rapid loss of plasma, and this prescription should clearly specify the type, amount, infusion rate, and infusion time (before, during, or after the procedure). Furthermore, Zubair ${ }^{8}$ suggested that a phlebotomy prescription should contain the following elements: the patient's name, the diagnosis, a date of birth or medical record number, the laboratory tests that should be performed, hematocrit levels, post-phlebotomy care instructions, and the target blood volume. However, a volume of $>2$ units ( $>1 \mathrm{~L})$ should be cleared by the requesting physician, and no more than 1-2 units should be removed during a 24-hour period. Moreover, before initiating the phlebotomy, Zubair ${ }^{8}$ suggested completing the following evaluations: blood pressure, pulse, respiration, temperature, hematocrit levels, and an arm inspection. ${ }^{8}$

For patients who require several rounds of phlebotomy, a catheter may be inserted to collect the blood, although only peripheral blood should be removed during each round. In these cases, $20 \mathrm{G}$ or $18 \mathrm{G}$ needles are needed to allow for adequate blood flow. However, the risk of air embolism from vacuum tubes is being debated, ${ }^{36-38}$ as the most common complication is a reduced blood flow rate because of venous obstruction during the suction. ${ }^{39}$ The required level of site-specific sterilization is similar to that for peripheral catheter insertion, ${ }^{9}$ although very strict sterilization may not be required, as the collected blood is not reinjected. Therefore, the optimal technique involves insertion of the phlebotomy catheter immediately before the procedure and removal immediately after the procedure, in order to minimize the risk of vein damage.

Patients with chronic hemolytic anemia and iron overload may not tolerate phlebotomy, and both sound clinical judgment and careful monitoring are essential for managing patients who are undergoing therapeutic phlebotomy. Furthermore, it is important to encourage patients to drink fluids before and after each treatment, and it may be helpful to instruct patients to avoid strenuous physical activities for 24 hours after each treatment. ${ }^{8}$ After the phlebotomy, the patient should lean back and rest quietly for a few minutes, and then slowly return to an upright position. Once the patient is able to walk, he or she can be transferred to a monitoring area and have a light meal with liquids, although alcoholic or caffeinated beverages should be avoided for several hours after the phlebotomy. Moreover, patients should not smoke for $>30$ minutes after the phlebotomy. These recommendations should be clearly stated in the treatment guidelines at the relevant medical institution, and patient compliance should be carefully recorded in the patient's clinical records. ${ }^{35}$ 
Phlebotomy is associated with several rare adverse events, such as thrombosis, although the risk of post-phlebotomy thrombosis is increased in patients with polycythemia vera. ${ }^{9}$ The most common adverse event is the development of a hematoma at the drawing site..$^{35}$ The hematoma is mild in most cases, although severe cases may involve damage to the surrounding tissues, nerves, and blood vessels. The risk of a hematoma can be reduced by applying pressure to the drawing site, and pressure and ice should be used to slow the bleeding if a hematoma occurs. Another common adverse effect is syncope, ${ }^{9}$ which can be recognized by several symptoms, such as loss of strength, sweat, dizziness, and pallor; these symptoms can progress to loss of consciousness or even convulsions. If the patient faints during a phlebotomy, the tourniquet should be immediately released, the needle should be removed or the catheter should be capped and appropriate treatment should be used to manage the symptoms. The rapid decrease in blood volume can also be accompanied by nausea and vomiting, and the phlebotomy should be interrupted to provide appropriate treatment in cases of severe vomiting. However, antiemetic drugs are not generally recommended as a pretreatment for phlebotomy. ${ }^{9}$

Another complication of phlebotomy is needle-stick injuries among health-care workers (HCWs). Unfortunately, bloodborne pathogen exposure can result from percutaneous injuries (including needle-sticks), and these injuries are associated with a risk of HIV, hepatitis $\mathrm{B}$, hepatitis $\mathrm{C}$, and other pathogen transmissions to $\mathrm{HCWs}{ }^{40,41}$ In this context, an estimated 3.35 million HCWs experience a percutaneous injury with a contaminated sharp object every year. ${ }^{41}$ Furthermore, Kassa et a ${ }^{42}$ reported that 504 of 1,624 HCWs (31\%) had experienced needle or sharps injuries, and that phlebotomy and specimen handling accounted for $9 \%(12 / 132)$ of these exposures. Therefore, phlebotomists should be well acquainted with the possibility of needle-stick injuries and blood-borne pathogen exposure, and take appropriate measures to avoid these injuries and exposures. Moreover, we recommend that regulations or guidelines for phlebotomy should include precautions regarding needle-stick injuries, the immediate treatment steps, post-exposure prophylaxis (eg, serological testing to exclude infection), vaccination or medications, and a notification system to alert the appropriate infection control center.

\section{Conclusion}

In this review, we examined the therapeutic effects and use of phlebotomy as the recommended treatment for hemochromatosis, polycythemia vera, porphyria cutanea tarda, sickle cell disease, and NALFD with hyperferritinemia. In addition, phlebotomy can be considered as a component of treatment for patients with high serum iron levels. However, it is important to make treatment decisions based on each patient's condition and the relevant guidelines for prescribing and implementing phlebotomy. Furthermore, we recommend prioritizing the creation of treatment guidelines with staff and patient education programs for institutions that are seeking to introduce phlebotomy programs. These guidelines should also focus on patient care after the completion of the phlebotomy. In conclusion, the evidence that we have reviewed indicates that phlebotomy is safe and provides good efficacy with relatively low costs, although further studies are needed to improve the treatment guidelines for phlebotomy.

\section{Acknowledgments}

This work was supported by the Soonchunhyang University Research Fund. The authors thank Editage (www.editage.co.kr) for English language editing.

\section{Disclosure}

The authors report no conflicts of interest in this work.

\section{References}

1. Parapia LA. History of bloodletting by phlebotomy. Br J Haematol. 2008;143(4):490-495.

2. Schneeberg NG. A twenty-first century perspective on the ancient art of bloodletting. Trans Stud Coll Physicians Phila. 2002;24:157-185.

3. Estes JW. George Washington and the doctors: treating America's first superhero. Med Herit. 1985;1(1):44-57.

4. Sajid MI. Hijama therapy (wet cupping) - its potential use to complement British healthcare in practice, understanding, evidence and regulation. Complement Ther Clin Pract. 2016;23:9-13.

5. El Sayed SM, Mahmoud HS, Nabo MMH. Medical and scientific bases of wet cupping therapy (Al-hijamah): in light of modern medicine and prophetic medicine. Altern Integ Med. 2013;2:122.

6. Cao H, Han M, Zhu X, Liu J. An overview of systematic reviews of clinical evidence for cupping therapy. JTradit Chin Med Sci. 2015;2(1): 3-10.

7. Assi TB, Baz E. Current applications of therapeutic phlebotomy. Blood Transfus. 2014;12(suppl 1):s75-s83.

8. Zubair A. Therapeutic phlebotomy. Clin Liv Dis. 2014;4(5):102-106.

9. Cook LS. Therapeutic phlebotomy: a review of diagnoses and treatment considerations. J Infus Nurs. 2010;33(2):81-88.

10. Bose N, Kanzariya H. Role of therapeutic apheresis and phlebotomy techniques in anaesthesia and critical care. Indian J Anaesth. 2014;58(5):672-678.

11. Marchioli R, Finazzi G, Specchia G, Masciulli A, Mennitto MR, Barbui T. The CYTO-PV: a large-scale trial testing the intensity of CYTOreductive therapy to prevent cardiovascular events in patients with polycythemia vera. Thrombosis. 2011;2011:9.

12. Marchioli R, Finazzi G, Specchia G, et al; CYTO-PV Collaborative Group. Cardiovascular events and intensity of treatment in polycythemia vera. N Engl J Med. 2013;368(1):22-33.

13. Bornstein R, Menon D, York E, Sproule B, Zak C. Effects of venesection on cerebral function in chronic lung disease. Can J Neurol Sci. 1980;7(4):293-296.

14. Chetty KG, Light RW, Stansbury DW, Milne N. Exercise performance of polycythemic chronic obstructive pulmonary disease patients: effect of phlebotomies. Chest. 1990;98(5):1073-1077.

15. Piccirillo G, Fimognari FL, Valdivia JL, Marigliano V. Effects of phlebotomy on a patient with secondary polycythemia and angina pectoris. Int J Cardiol. 1994;44(2):175-177. 
16. Warnes CA, Williams RG, Bashore TM, Child JS, Connolly HM, Dearani JA. ACC/AHA 2008 Guidelines for the management of adults with congenital heart disease: a report of the American College of Cardiology/American Heart Association Task Force on Practice Guidelines (writing committee to develop guidelines on the management of adults with congenital heart disease). Circulation. 2008;118(23):e714-e833.

17. Andersen RV, Tybjaerg-Hansen A, Appleyard M, Birgens H, Nordestgaard BG. Hemochromatosis mutations in the general population: iron overload progression rate. Blood. 2004;103(8):2914-2919.

18. Adams PC, Barton JC. How I treat hemochromatosis. Blood. 2010;116(3):317-325.

19. Bacon BR, Adams PC, Kowdley KV, Powell LW, Tavill AS. Diagnosis and management of hemochromatosis: 2011 practice guideline by the American Association for the Study of Liver Diseases. Hepatology. 2011;54(1):328-343.

20. Ramsy CA, Jagnus IA, Turnbull A, Baker $\mathrm{H}$. The treatment of porphyria cutanea tarda by venesection. $Q J$ Med. 1974;43(169):1-24.

21. Rocchi E, Gibertini P, Cassanelli M, Pietrangelo A, Borghi A, Ventura E. Serum ferritin in the assessment of liver iron overload and iron removal therapy in porphyria cutanea tarda. J Lab Clin Med. 1986;107(1): $36-42$.

22. ClinicalTrials.gov [webpage on the Internet]. Erythrocyte Apheresis Versus Phlebotomy in Hemochromatosis. 2007. Available from: http://www. clinicaltrials.gov/ct2/show/NCT00509652. Accessed July 27,2007.

23. Bouchaïr N, Manigne P, Kanfer A, et al. Prevention of sickle cell crises with multiple phlebotomies. Arch Pediatric. 2000;7(3):249-255.

24. Rombos Y, Tzanetea R, Kalotychou V, et al. Amelioration of painful crises in sickle cell disease by venesections. Blood Cells Mol Dis. 2002;28(2):283-287.

25. Aygun B, Mortier NA, Kesler K, et al. Stroke with transfusions changing to hydroxyurea (SWiTCH) trial investigators. Therapeutic phlebotomy is safe in children with sickle cell anaemia and can be effective treatment for transfusional iron overload. Br J Haematol. 2015;169(2):262-266.

26. Summarell CC, Sheehan VA. Use of hydroxyurea and phlebotomy in pediatric patients with hemoglobin sickle cell disease. Exp Biol Med (Maywood). 2016;241(7):737-744.

27. Byrne CD, Targher G. NAFLD: a multisystem disease. J Hepatol. 2015;62(1 suppl):S47-S64.

28. Milic S, Mikolasevic I, Krznaric-Zrnic I, et al. Nonalcoholic steatohepatitis: emerging targeted therapies to optimize treatment options. Drug Des Devel Ther. 2015;9:4835-4845.
29. Koplay M, Sivri M, Erdogan H, Nayman A. Importance of imaging and recent developments in diagnosis of nonalcoholic fatty liver disease. World J Hepatol. 2015;7(5):769-776.

30. Marchesini G, Bugianesi E, Forlani G, et al. Nonalcoholic fatty liver, steatohepatitis, and the metabolic syndrome. Hepatology. 2003;37(4):917-923.

31. Valenti L, Fracanzani AL, Dongiovanni P, et al. A randomized trial of iron depletion in patients with nonalcoholic fatty liver disease and hyperferritinemia. World J Gastroenterol. 2014;20(11):3002-3010.

32. Dongiovanni P, Fracanzani AL, Fargion S, Valenti L. Iron in fatty liver and in the metabolic syndrome: a promising therapeutic target. J Hepatol. 2011;55(4):920-932.

33. Adams LA, Crawford DH, Stuart K, et al. The impact of phlebotomy in nonalcoholic fatty liver disease: a prospective, randomized, controlled trial. Hepatology. 2015;61(5):1555-1564.

34. Ahfsdruginformation.com [homepage on the Internet]. Bethesda: AHFS Drug Information $^{\circledR}$; c2009-10[updated 2015 Nov 17; cited 2015 Dec 3]. Available from: http://www.ahfsdruginformation.com/. Accessed December 29, 2015.

35. Roback J, editor. Technical Manual. 16th ed. Arlington, VA: AABB; 2008.

36. Jahangiri M, Rayner A, Keogh B, Lincoln C. Cerebrovascular accident after vacuum-assisted venous drainage in a Fontan patient: a cautionary tale. Ann Thorac Surg. 2001;72(5):1727-1728.

37. Lotterle J, Schellmann B. Unbekannte Gefahr bei Adelaß mit Unterdruckflaschen: Luftembolie [Unknown danger in phlebotomy with vacuum flasks: air embolism]. Zeitschrift fur Rechtsmedizin. 1980;5(4):247-254. German.

38. Siegel P, Petrides PE. Congenital and acquired polycythemias. Deutsches Aerzteblatt Int. 2008;105(4):62-68.

39. Iron Disorders Institute. Therapeutic Phlebotomy Treatment for Iron Overload. Greenville, SC: Iron Disorders Institute; 2004:3-54.

40. Kessler CS, McGuinn M, Spec A, Christensen J, Baragi R, Hershow RC. Underreporting of blood and body fluid exposures among health care students and trainees in the acute care setting: a 2007 survey. $\mathrm{Am}$ J Infect Control. 2011;39(2):129-134.

41. Prüss-Ustün A, Rapiti E, Hutin Y. Estimation of the global burden of disease attributable to contaminated sharps injuries among health-care workers. Am J Ind Med. 2005;48(6):482-490.

42. Kassa G, Selenic D, Lahuerta M, et al. Occupational exposure to bloodborne pathogens among health care workers in Botswana: reporting and utilization of postexposure prophylaxis. Am J Infect Control. Epub 2016 Mar 24.
Journal of Blood Medicine

\section{Publish your work in this journal}

The Journal of Blood Medicine is an international, peer-reviewed, open access, online journal publishing laboratory, experimental and clinical aspects of all aspect pertaining to blood based medicine including but not limited to: Transfusion Medicine; Blood collection, Donor issues, Transmittable diseases, and Blood banking logistics; Immunohematology; Artificial and alternative

\section{Dovepress}

blood based therapeutics; Hematology; Biotechnology/nanotechnology of blood related medicine; Legal aspects of blood medicine; Historical perspectives. The manuscript management system is completely online and includes a very quick and fair peer-review system. Visit http://www.dovepress.com/ testimonials.php to read real quotes from published authors. 